\title{
TRANSMEMBRANE HELIX AND TOPOLOGY PREDICTION USING HIERARCHICAL SVM CLASSIFIERS AND AN ALTERNATING GEOMETRIC SCORING FUNCTION
}

\author{
Allan Lo ${ }^{1,2}$, Hua-Sheng Chiu ${ }^{3}$, Ting-Yi Sung ${ }^{3}$, Wen-Lian $\mathrm{Hsu}^{3, *}$ \\ ${ }^{1}$ Bioinformatics Program, Taiwan International Graduate Program, Academia Sinica, Taipei, Taiwan \\ ${ }^{2}$ Department of Life Sciences, National Tsing Hua University, Hsinchu, Taiwan \\ ${ }^{3}$ Bioinformatics Lab., Institute of Information Science, Academia Sinica, Taipei, Taiwan \\ Email: \{allanlo,huasheng, tsung,hsu\}@iis.sinica.edu.tw
}

\begin{abstract}
Motivation: A key class of membrane proteins contains one or more transmembrane (TM) helices, traversing the membrane lipid bilayer. Various properties such as the length, arrangement and topology or orientation of TM helices, are closely related to a protein's functions. Although a range of methods have been developed to predict TM helices and their topologies, no single method consistently outperforms the others. In addition, topology prediction has much lower accuracy than helix prediction, and thus requires continuous improvements. Results: We develop a method based on support vector machines (SVM) in a hierarchical framework to predict TM helices first, followed by their topology. By partitioning the prediction problem into two steps, specific input features can be selected and integrated in each step. We also propose a novel scoring function for topology models based on membrane protein folding process. When benchmarked against other methods in terms of performance, our approach achieves the highest scores at $86 \%$ in helix prediction $\left(Q_{2}\right)$ and $91 \%$ in topology prediction $(T O P O$ ) for the high-resolution data set, resulting in an improvement of $6 \%$ and $14 \%$ in their respective categories over the second best method. Furthermore, we demonstrate the ability of our method to discriminate between membrane and non-membrane proteins, with higher than $99 \%$ in accuracy. When tested on a small set of newly solved structures of membrane proteins, our method overcomes some of the difficulties in predicting TM helices by incorporating multiple biological input features.
\end{abstract}

\section{INTRODUCTION}

Integral membrane proteins constitute a wide and important class of biological entities that are crucial for life, representing about $25 \%$ of the proteins encoded by several genomes ${ }^{1-3}$. They also play a key role in various cellular processes including signal and energy transduction, cell-cell interactions, and transport of solutes and macromolecules across membranes ${ }^{4}$. Despite their biological importance, the proportion of available highresolution structures is exceedingly limited at about $0.5 \%$ of all solved structures ${ }^{5}$, compared to that of globular proteins deposited in the Protein Data Bank $(\mathrm{PDB})^{6}$. In the absence of a high-resolution structure, an accurate structural model is important for the functional annotation of membrane proteins. A membrane protein structural model defines the number and location of transmembrane helices (TMHs) and the orientation or topology of the protein relative to the lipid bilayer. However, experimental approaches for identifying membrane protein structural models are timeconsuming ${ }^{7}$. Therefore, bioinformatics development in sequence-based prediction methods is valuable for elu- cidating the structural genomics of membrane proteins.

Many different methods have been developed to predict structural models of transmembrane helix (TMH) proteins. Earlier approaches relied on physico-chemical properties such as hydrophobicity ${ }^{8-10}$ to identify TMH regions. Recently, more advanced methods using hidden Markov models ${ }^{3,11}$ and neural networks ${ }^{12}$ have been developed, and they have achieved significant improvements in prediction accuracy. Although several methods are available, none of them have integrated multiple biological input features in a machine-learning framework. Furthermore, an evaluation study ${ }^{13}$ concluded that current accuracies were over-estimated, and topology prediction remained a major challenge.

In this paper, we propose a machine-learning approach called SVMtmh (SVM for transmembrane helix prediction) in a hierarchical classification framework to predict membrane protein structure. We divide the prediction task into two successive steps by using a tertiary classifier consisting of two hierarchical binary classifiers. The number and location of TMHs are predicted in the first step, followed by the prediction of the topology in the second step.

\footnotetext{
${ }^{*}$ Corresponding author.
} 
Our key contributions are as follows: 1) By decomposing the prediction into two steps, we reduce the complexity involved in each step, and biological input features relevant to each classifier can be applied. 2) We select multiple input features, including those based on different structural parts of a TMH protein, and integrate them to predict helices. 3) For topology prediction, we propose a novel topology scoring function based on the current understanding of membrane protein insertion. To the best of our knowledge, the proposed topology scoring function is the first model to capture the relationship between topogenic factors and topology formation.

The performance of SVMtmh is compared with other methods across several benchmark data sets and SVMtmh achieves a marked improvement in both helix and topology prediction. Specifically, SVMtmh achieves the highest score at $91 \%$ for topology prediction $(T O P O)$ and $86 \%$ for helix prediction $\left(Q_{2}\right)$ in the high-resolution data set, an improvement of $14 \%$ and $6 \%$, respectively, compared to the second highest score. In addition, SVMtmh yields the lowest false positive rate at $0.5 \%$ when tested for discrimination between membrane and non-membrane proteins. Finally, we apply SVMtmh to analyze a newly solved structure of bacteriorhodopsin (bR) and show that our method can provide the correct structural model which is in close agreement with the structure obtained through X-ray crystallography. We also provide a detailed analysis of the comparison with other methods and conclude with a summary and directions for future work.

\section{METHODS}

\subsection{System architecture}

The proposed approach uses hierarchical binary classifiers to predict the helices and topology of an integral membrane protein. We represent the problem of membrane protein structure prediction as a multiple classification process and solve it in two steps using hierarchical SVM classifiers. The overall framework is described in this section.

Each residue of a TMH protein can be regarded as belonging to one of the three classes defined by its position with respect to the membrane: inner $(i)$ loop, transmembrane helix $(H)$, and outer $(o)$ loop. The aim of predicting membrane protein structures is to identify the correct class of each residue. Since there are three classes for a protein sequence, we design a tertiary classifier, which consists of two binary classifiers in a hierarchical structure. An overview of the system architecture is shown in Fig. 1.

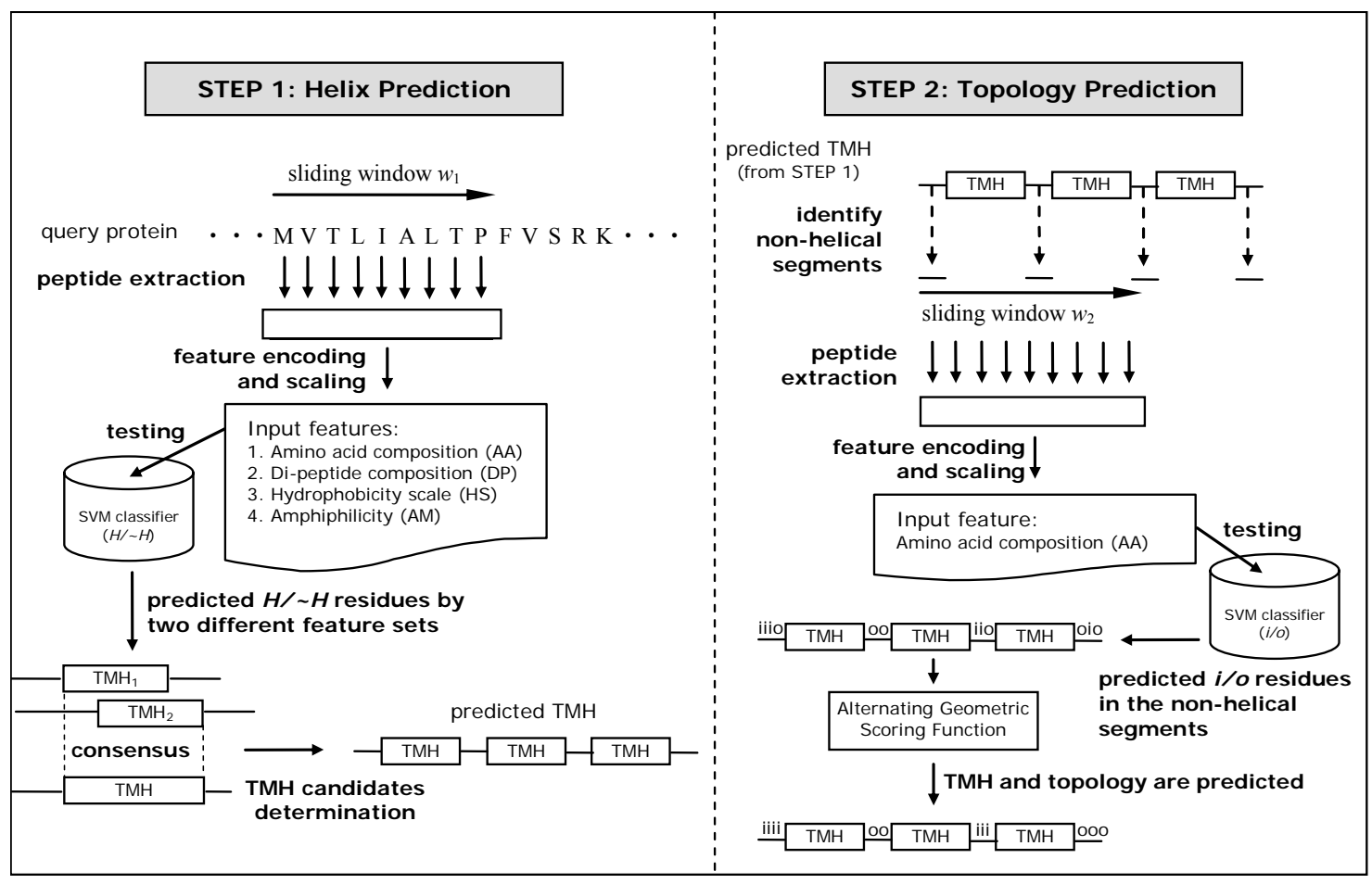

Fig. 1. Overview of the SVMtmh system architecture. 
In Step 1, TM and non-TM residues $(H / \sim H)$ are predicted. We use different feature sets (Section 3.3) to train our SVM classifiers and then combine the results from the best two combinations into a consensus prediction, which is screened for TMH candidates and subsequently assembled into physical TMH segments. In Step 2, the remaining non-helix residues $(\sim H)$ from Step 1 are classified as either inner or outer $(i / o)$ residues. To determine if the topology of the protein is an inner $(i)$ or an outer loop $(o)$, we apply the proposed alternating geometric scoring function. Briefly, the classification framework is performed in two steps, each of which uses an associated binary classifier $(H / \sim H, i / o)$.

We use sliding windows to partition a protein sequence into peptides. The optimal length of the sliding window, $w$, is incrementally searched from 3 to 41 for both classifiers. The optimal window sizes, $w_{l}$ for the first classifier and $w_{2}$ for the second classifier, are found to be 21 and 29 , respectively.

\subsection{Training and testing}

We train our classifiers with the LIBSVM package ${ }^{14}$ and Radial Basis Function (RBF) is chosen as the kernel function. The associated parameters $(C, \gamma)$ are optimized at $(1.8661,0.1250)$. The cost weight is adjusted to avoid under-prediction in unbalanced data sets. Since the helix and non-helix classes make up about $30 \%$ and $70 \%$ of the data set respectively, we set the cost weight at $7 / 3$ for the first classifier. Similarly, we set the cost weight at $1 / 1$ for the second classifier to reflect the proportion of the inner and outer loop classes in the data set. Ten-fold cross-validation is used to evaluate our method. The data set is first divided into ten subsets of equal size. Each subset is in turn tested using the classifier trained on the remaining nine subsets. Since each residue of the whole data set is only predicted once, the overall prediction accuracy is the percentage of correctly predicted residues. The values in the feature vectors are scaled in the range of $[0,1]$.

\subsection{Helix prediction}

\subsubsection{Feature selection and extraction}

The choice of relevant features is critical in any prediction models. Thus, in the present study we select features that capture important relationships between a sequence and the structure. TMH proteins are subject to global constraints of the lipid bilayer since they contain membrane-spanning helices ${ }^{15}$. Additionally, TM helices can be divided into distinct local structural parts, including the core and end regions based on the propensity of amino acids ${ }^{3}$. Fig. 2 shows the selection of features to capture both the global and local information of a TM helix. The representation of each feature is described below:
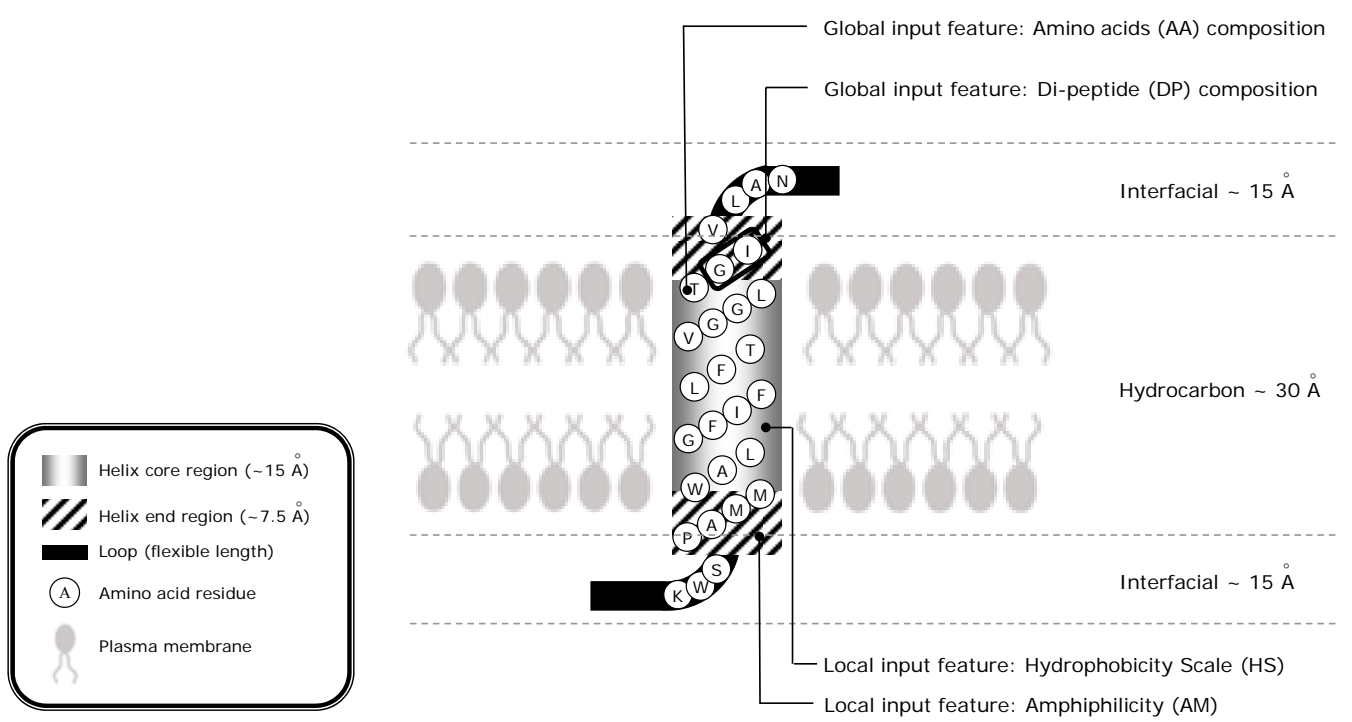

Fig. 2. Transmembrane (TM) helix structure in the lipid bilayer: helix core and end regions. Loops connect between the adjacent TM helices. We select global and local input features to capture information contained in a TM helix. Global input features: amino acid (AA) and di-peptide (DP compositions. Local input features: hydrophobicity scale (HS) ${ }^{16}$ and amphiphilicity (AM) $)^{17}$. The helix core region is surrounded by an aliphatic hydrocarbon layer about $30 \AA$ in thickness. The helix end regions are embedded in the water-membrane interface of about $15 \AA$. 
1. Amino acid composition (AA): This basic feature enables us to capture the global information of a TM helix. Each residue of a peptide is represented by a vector of length of 20 which is indicated by 1 in the position corresponding to the amino acid type of the residue, and 0 otherwise.

2. Di-peptide composition $(D P)$ : We consider the coupling effect of two adjacent residues that contain global information along the sequence. This feature is represented by the pair-residue occurrence probability, $\mathrm{P}(\mathrm{X}, \mathrm{Y})$ where $(\mathrm{X}, \mathrm{Y})$ is an ordered pair of amino acids of $X$ followed by $Y$. The vector space of this feature input comprises 400 dimensions.

3. Helix core feature: Hydrophobicity is used to capture local information within the core region of a TM helix where it is a major stabilizing factor ${ }^{16}$. We select a hydrophobicity scale (HS) recently determined by membrane insertion experiments ${ }^{16}$. Each residue is represented by a vector of length 20 that has a real value corresponding to its hydrophobicity.

4. Helix ends feature: The end regions of a TM helix near the membrane-water interface exhibit a preference for aromatic and polar residues, as shown in amino acid propensity studies ${ }^{16,17}$. We select an amphiphilicity (AM) index ${ }^{17}$ as a input feature to capture the local information contained in the helixcapping ends. Each residue is represented by a vector of length 20 that has a real value corresponding to its amphiphilicity.

\subsubsection{Determination of $\mathrm{TMH}$ candidates}

To identify potential TMH regions, it is necessary to determine if there are any TMH candidates among our initial prediction results. We do this by modifying the algorithm proposed in the THUMBUP program $^{18}$ to determine TMH candidates and assemble them into physical TMH segments.

\section{Step 1: Filtering}

We define a cut-off value, $l_{\text {min }}$, as the minimal length for a TMH candidate. A predicted helix segment is a TMH candidate if its length is at least $l_{\text {min }}$; otherwise, it is converted to a non-helix segment. Steps 2 and 3 describes the assembly of a TMH candidate.

\section{Step 2: Extension}

An optimal TMH length, $l_{\text {opt }}$, is set at 21 to reflect the thickness of the hydrocarbon core of a lipid bilayer ${ }^{19}$. If the length of a TMH candidate is between $l_{\min }$ and $l_{\text {opt }}$, it is extended to $l_{\text {opt }}$ from its $\mathrm{N}$ - and C-termini. Two or more $\mathrm{TMH}$ candidates are merged if they overlap after the extension.

\section{Step 3: Splitting}

We define $l_{\max }$, as the cut-off value for the length of a $\mathrm{TMH}$ candidate to be split. A TMH candidate whose length is greater than or equal to $l_{\max }$ is split into two helices, starting from its $\mathrm{N}$ - and C-termini with the loop in the center.

We optimize $l_{\min }$ and $l_{\max }$ on the training data set (Section 3.1). The optimized values for $l_{\min }$ and $l_{\max }$ for the best prediction performance are 9 and 38, respectively.

\subsection{Topology prediction}

\subsubsection{Input feature}

Using the second classifier, we predict the topology label $(i / o)$ of each non-helix residues from the results of the first classifier $(H / \sim H)$. Amino acid composition is employed as the input feature. The encoding scheme follows the same procedure outlined in the helix prediction section.

\subsubsection{Alternating geometric scoring function}

The purpose of predicting of the topology of a TMH protein is to determine the orientation of the protein with respect to the membrane. A TMH protein follows special constraints on its topology such that it always starts with an inner $(i)$ loop or outer $(o)$ loop that must alternate in order to connect the TM helices. Therefore, the problem of predicting the topology of a TMH protein is reduced to predicting the topology of the first loop located at the N-terminus.

There is growing body of evidence that the final topology is influenced by multiple signals distributed along the entire protein in the loop segments, including the charge bias, loop size, and folding of the N-terminal loop domain ${ }^{20}$. Furthermore, the widely accepted twostage model for membrane protein folding suggests that the final topology of a membrane protein is established in the early stages of membrane insertion ${ }^{21}$. These biological phenomena form the basis of our assumptions about topology models. First, we assume that topology formation is a result of contributing signals present in 
the various loop segments. Second, signals embedded in the loop segments near the $\mathrm{N}$-terminus are more likely to be a factor in the formation of topology since they are inserted in the membrane at an earlier time. Based on these assumptions, we develop a novel topology scoring function that considers the topogenic contribution from all loop segments that diminishes over a distance away from the N-terminus.

In the proposed topology scoring function, the contribution of signals in the loop segments varies inversely proportional to their distance from the $\mathrm{N}$-terminus in a geometric series: Given a transmembrane protein that has $n$ non-helical segments $s_{j}(1 \leq j \leq n$ and $n, j \in \mathbb{N})$ predicted in the first step: For each $s_{j}$ of length $\left|s_{j}\right|$, we define two ratios, $R_{i}$ and $R_{o}$, to represent the predicted ratios of topology labels $i$ and $o$, respectively.

$$
\begin{aligned}
& R_{i}(j)=\left(\# \text { of "inside" residues } /\left|s_{j}\right|\right) \times 100 \% \\
& R_{o}(j)=\left(\# \text { of "outside" residues } /\left|s_{j}\right|\right) \times 100 \%,
\end{aligned}
$$

where $R_{i}+R_{o}=100 \%$. To determine the protein topology, we define two topology scores, $T S_{i}$ and $T S_{o}$, where $T S_{i}$ is for the N-terminal loop on the inside of membrane and $T S_{o}$ is for the outside.

$$
\begin{gathered}
T S_{i}=\sum_{1 \leq j \leq n} W(j) \times\left[\alpha R_{i}(j)+(1-\alpha) R_{o}(j)\right] \\
T S_{o}=\sum_{1 \leq j \leq n} W(j) \times\left[(1-\alpha) R_{i}(j)+\alpha R_{o}(j)\right] \\
\alpha=\left\{\begin{array}{l}
1, \text { if } j \text { is odd } \\
0, \text { if } j \text { is even }
\end{array}\right. \\
W(j)=1 / b^{(j-1) \times E I}, b \text { and } E I \in \mathbb{R},
\end{gathered}
$$

where $b$ and $E I$ denote the base and the exponent increment, respectively. $W(j)$ is a geometric function which assigns weights to the $R_{i}(j)$ and $R_{o}(j)$ terms. If $T S_{i} \geq T S_{o}$, then the topology of the N-terminal loop is inside; otherwise, the topology is outside.

For the calculation of topology scores, the geometric scoring function alternates between the inner $(i)$ and outer $(o)$ loops to take into account the alternating nature of the connecting loops. Fig. 3 illustrates the calculation of alternating geometric scoring function for an example protein.

\section{RESULTS AND DISCUSSION}

\subsection{Data sets}

1. Low-resolution TMH proteins: We train and perform ten-fold cross-validation on a collection of low-resolution data set compiled by Möller et $a l^{22}$. We select 145 proteins of good reliability from a set of 148 non-redundant proteins. We manually validate this data set using annotations from SWISS-PROT release $49.0^{23}$ and further remove two proteins because they have no membrane protein annotations. The final data set contains 143 proteins for which low-resolution topology models are available. This entire data set is also used to train our model for testing on the following three data sets.

2. High-resolution TMH proteins: We use a collection of 36 high-resolution TMH proteins from PDB compiled by Chen et al. ${ }^{13}$ and obtain topology information for 35 out of 36 proteins. We validate this data set using annotations from SWISS-PROT release $49.0^{23}$ and update the topologies of two proteins.

3. Soluble proteins: A collection of 616 highresolution soluble proteins from PDB compiled by Chen et $_{\text {al }}{ }^{13}$ is used to test for discrimination between membrane and soluble proteins.

4. Newly solved TMH proteins: Four newly solved high-resolution TMH proteins ${ }^{24}$ are used as an independent test set.

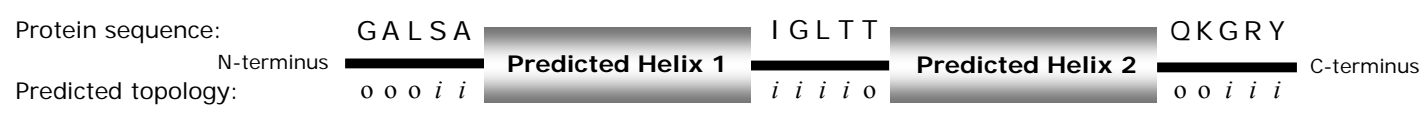

Fig 3. An example of evaluating a TMH protein's topology with alternating geometric scoring function. Helices are predicted in the first step (Section 2.3). A predicted loop segment can have more than one type of topology. We use the proposed alternating geometric scoring function to determine the final topology. In this example, $R_{i}(1)=2 / 5, R_{o}(1)=3 / 5, R_{i}(2)=4 / 5, R_{o}(2)=1 / 5, R_{i}(3)=3 / 5$ and $R_{o}(3)=2 / 5$. Given a set of optimal values for $(b, E I)=(1.6,1.0)$ indicated in Section 3.6, $T S_{i}=1 \times R_{i}(1)+1 /\left(1.6^{1.0}\right) \times R_{o}(2)+1 /\left(1.6^{2.0}\right) \times R_{i}(3) \fallingdotseq 0.7594$. Similarily, $T S_{o} \fallingdotseq 1.2563$. $T S_{o}>T S_{i}$, therefore, the final topology for the N-terminal loop is outside $(o)$. 


\subsection{Evaluation metrics}

There are two sets of evaluation measures for the TMH prediction: per-segment and per-residue accuracies ${ }^{13}$. Per-segment scores indicate how accurately the location of a $\mathrm{TMH}$ region is predicted and per-residue scores report how well each residue is predicted. Table 1 lists the per-segment and per-residue metrics used in this paper.

In the calculation of per-segment scores, two issues must be addressed when counting a helix as correctly predicted. First, a minimal overlap of observed helix segments must be defined. For this, we use a less relaxed criterion which requires at least 9 overlapping residues. An evaluation study by Chen et al. ${ }^{13}$ used a more relaxed minimal overlap of only 3 residues. Second, we do not allow an overlapping observed helix to be counted twice. We use the following examples to illustrate these two issues $(\mathrm{H}=$ Helix $)$ :

Observation: - - HHHHHHHHHHHHH - - - HНHНHНHHHH -

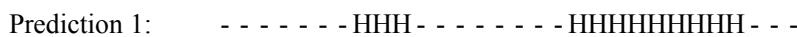
Prediction 2: - - - HHHHHHHHHHHHHHHHHHHHHHH - - Prediction 3: - - HHH - HHHHHHHHH - - - HHHHHHHHH - - -

Prediction 1 achieves $100 \%$ accuracy if the minimal overlap is 3 residues. If the minimal overlap is 9 resi- dues, Prediction 1 achieves 50\% accuracy. Prediction 2 achieves $50 \%$ accuracy because it already overlaps with the first observed helix. Prediction 3 achieves 100\% accuracy if the minimal overlap is 3 residues, but the second predicted helix is an over-prediction since we only count an overlapping observed helix only once. Prediction 3 achieves $50 \%$ accuracy if the minimal overlap is 9 residues because the first predicted helix does not satisfy the minimal overlap requirement. In addition, the second predicted helix is also an overprediction, thus it is not counted.

\subsection{Performance of input feature combinations for helix prediction}

We test the performance of different input feature combinations for the first classifier. The following combinations are considered: 1) AA only; 2) AA and any one of DP, HS, and AM; 3) AA and any two of DP, HS, and AM; and 4) all four features. We also construct a consensus prediction from the two top-performing combinations through probability estimation using LIBSVM ${ }^{25}$. The value of the estimated probability for each residue corresponds to the confidence given for its predicted class. In the case of disagreement between the predicted classes, the consensus prediction takes the result of a prediction that has the highest probability.

Table 1. Evaluation metrics used in this work. Per-segment metrics include $Q_{o k}, Q_{h t m}^{\%}, Q_{h t m}^{\% \text { prd }}$ and TOPO. Per-residue metrics include $Q_{2}$, $Q_{2 T}^{\%}$, ans $Q_{2 T}^{\%}$. prd $N_{\text {prot }}$ is the number of proteins in a data set. We follow the same performance measures proposed by Chen et al. ${ }^{13}$

\begin{tabular}{|c|c|c|}
\hline Symbol & Formula & Description \\
\hline$Q_{o k}$ & $\frac{\sum_{i}^{N_{\text {prot }}} \delta_{i}}{N_{\text {prot }}} \times 100 \%$, with $\delta_{i}=\left\{\begin{array}{l}1, \text { if } Q_{h t m}^{\% \text { obs }} \wedge Q_{h t m}^{\% \text { prd }}=100 \% \text { for protein } i \\
0, \text { otherwise }\end{array}\right.$ & $\begin{array}{l}\text { percentage of proteins in which all its } \\
\text { TMH segments are predicted correctly }\end{array}$ \\
\hline$Q_{h t m}^{\% o b s}$ & $\frac{\text { number of correctly predicted TM in data set }}{\text { number of TM observed in data set }} \times 100 \%$ & TMH segment recall \\
\hline$Q_{h t m}^{\% \text { prd }}$ & $\frac{\text { number of correctly predicted TM in data set }}{\text { number of TM predicted in data set }} \times 100 \%$ & TMH segment precision \\
\hline TOPO & $\frac{\text { number of proteins with correctly predicted topology }}{N_{\text {prot }}} \times 100 \%$ & percentage of correctly predicted topology \\
\hline$Q_{2}$ & $\frac{\sum_{i}^{N_{\text {prot }}} \frac{\text { number of residues predicted correctly in protein } i}{\text { number of residues in prtoein } i}}{N_{\text {prot }}} \times 100 \%$ & percentage of correctly predicted $\mathrm{TMH}$ residues \\
\hline$Q_{2 T}^{\% o b s}$ & $\frac{\text { number of residues correctly predicted in TM helices }}{\text { number of residues observed in TM helices }} \times 100 \%$ & $\mathrm{TMH}$ residue recall \\
\hline$Q_{2 T}^{\% p r d}$ & $\frac{\text { number of residues correctly predicted in TM helices }}{\text { number of residues predicted in TM helices }} \times 100 \%$ & TMH residue precision \\
\hline
\end{tabular}


Table 2 shows the performance of combinations of input features and the consensus prediction. Combination 5 achieves the highest score for $Q_{o k}$ at $71.9 \%$ and performs consistently well in other per-segment and per-residue measures. Combination 6 has a strikingly high $Q_{2 T}^{\% \text { obs }}$ score of $85.9 \%$. The purpose of consensus prediction is to maximize the benefits of both combinations. In fact, the consensus approach increases the $Q_{o k}$ score of Combination 6 by $1.5 \%$, while the $Q_{2 T}^{\% \text { obs s }}$ score only decreases by $0.3 \%$. Compared to Combination 5 , the consensus has a decrease in $Q_{o k}$ of $1.4 \%$, but an increase in $Q_{2 T}^{\% \text { obs }}$ of $3.8 \%$. In addition, the consensus approach also scores the highest for $Q_{2}$ at $89.1 \%$. The consensus approach is selected as our best model for comparison with other approaches.

\subsection{Performance on high- and low- resolution data sets}

SVMtmh is compared to other methods for high and low-resolution data sets in Table 3. For the lowresolution set, SVMtmh ranks the highest among all the compared methods for per-segment measures in TOPO, $Q_{o k}$, and $Q_{h t m}^{\% \text { pred }}$ at $84 \%, 71 \%$, and $95 \%$, respectively. Specifically, SVMtmh improves TOPO by $5 \%$ over the second best method for the low-resolution data set. For the high-resolution set, most notably, SVMtmh has the highest score at $91 \%$ for TOPO, a $14 \%$ improvement over the second best method. Another marked improvement is also observed for the high-resolution set in

Table 2. Performance of input feature combinations and the consensus method. Input features: AA (amino acid composition), DP (di-peptide composition), HS (hydrophobicity scale) ${ }^{16}$ and AM (amphiphilicity $)^{17}$.

\begin{tabular}{|c|c|c|c|c|c|c|c|}
\hline \multirow{2}{*}{ No. } & \multirow{2}{*}{ Input Feature (s) } & \multicolumn{3}{|c|}{ Per-segment (\%) } & \multicolumn{3}{|c|}{ Per-residue (\%) } \\
\hline & & $Q_{o k}$ & $Q_{h t m}^{\% \text { obs }}$ & $Q_{h t m}^{\% p r d}$ & $Q_{2}$ & $Q_{2 T}^{\% \text { obs }}$ & $Q_{2 T}^{\% \text { prd }}$ \\
\hline 1 & AA & 71.2 & 93.8 & 93.9 & 89.1 & 82.9 & 83.0 \\
\hline 2 & $\mathrm{AA}+\mathrm{DP}$ & 69.8 & 94.0 & 93.8 & 88.9 & 81.9 & 83.2 \\
\hline 3 & $\mathrm{AA}+\mathrm{HS}$ & 71.2 & 92.8 & 94.2 & 89.1 & 81.9 & 84.0 \\
\hline 4 & $\mathrm{AA}+\mathrm{AM}$ & 70.5 & 93.6 & 93.6 & 89.1 & 83.0 & 82.9 \\
\hline 5 & $\mathrm{AA}+\mathrm{DP}+\mathrm{HS}$ & 71.9 & 93.6 & 94.2 & 89.0 & 81.8 & 83.7 \\
\hline 6 & $\mathrm{AA}+\mathrm{DP}+\mathrm{AM}$ & 69.0 & 93.4 & 94.0 & 89.0 & 85.9 & 80.6 \\
\hline 7 & $\mathrm{AA}+\mathrm{HS}+\mathrm{AM}$ & 68.3 & 93.3 & 94.2 & 88.8 & 79.8 & 84.4 \\
\hline 8 & $\mathrm{AA}+\mathrm{DP}+\mathrm{HS}+\mathrm{AM}$ & 69.1 & 92.3 & 95.4 & 89.0 & 80.9 & 84.3 \\
\hline 9 & Consensus $(5+6)$ & 70.5 & 93.2 & 94.9 & 89.1 & 85.6 & 81.4 \\
\hline
\end{tabular}

Table 3. Performance of prediction methods for low- and high-resolution data sets. Per-segment and per-residue scores of all methods compared are taken from an evaluation by Chen et al. ${ }^{13}$. TOPO scores for the high-resolution data set are re-evaluated due to the update of topology information. The shaded area outlines the four top-performing methods. Note that we do not have cross-validation results for all other methods. Therefore, their accuracies might be over-estimated. In addition, we use a minimal overlap of 9 residues whereas Chen et al. ${ }^{13}$ used only 3 residues. Methods are sorted by their $Q_{o k}$ values for the low-resolution data set.

\begin{tabular}{|c|c|c|c|c|c|c|c|c|c|c|c|c|c|c|}
\hline \multirow{3}{*}{ Methods } & \multicolumn{7}{|c|}{ Low-resolution } & \multicolumn{7}{|c|}{ High-resolution } \\
\hline & \multicolumn{4}{|c|}{ Per-segment (\%) } & \multicolumn{3}{|c|}{ Per-residue (\%) } & \multicolumn{4}{|c|}{ Per-segment (\%) } & \multicolumn{3}{|c|}{ Per-residue (\%) } \\
\hline & $Q_{o k}$ & $Q_{h t m}^{\% o b s}$ & $Q_{h t m}^{\% \text { prd }}$ & TOPO & $Q_{2}$ & $Q_{2 T}^{\% \text { obs }}$ & $Q_{T}^{\% \text { prd }}$ & $Q_{o k}$ & $Q_{h t m}^{\% \text { obs }}$ & $Q_{h t m}^{\% p r d}$ & TOPO & $Q_{2}$ & $Q_{2 T}^{\% o b s}$ & $Q_{2 T}^{\% \text { prd }}$ \\
\hline SVMtmh & 71 & 93 & 95 & 84 & 89 & 86 & 81 & 83 & 96 & 98 & 91 & 86 & 82 & 90 \\
\hline TMHMM2 & 68 & 91 & 94 & 77 & 89 & 82 & 84 & 75 & 92 & 96 & 66 & 80 & 72 & 88 \\
\hline PHDpsiHtm08 & 67 & 95 & 94 & 67 & 89 & 87 & 77 & 84 & 99 & 98 & 57 & 80 & 76 & 83 \\
\hline НMМTOP2 & 66 & 94 & 93 & 79 & 90 & 85 & 83 & 83 & 99 & 99 & 77 & 80 & 69 & 89 \\
\hline PRED-TMR & 58 & 92 & 93 & & 90 & 78 & 86 & 61 & 84 & 90 & & 76 & 58 & 85 \\
\hline PHDhtm08 & 57 & 86 & 86 & 68 & 87 & 83 & 75 & 64 & 77 & 76 & 60 & 78 & 76 & 82 \\
\hline PHDhtm07 & 56 & 85 & 86 & 72 & 87 & 83 & 75 & 69 & 83 & 81 & 69 & 78 & 76 & 82 \\
\hline SOSUI & 49 & 88 & 86 & & 88 & 79 & 72 & 71 & 88 & 86 & & 75 & 66 & 74 \\
\hline TopPred 2 & 48 & 84 & 79 & 59 & 88 & 74 & 71 & 75 & 90 & 90 & 57 & 77 & 64 & 83 \\
\hline DAS & 39 & 93 & 81 & & 86 & 65 & 85 & 79 & 99 & 96 & & 72 & 48 & 94 \\
\hline Ben-Tal & 35 & 79 & 90 & & 87 & 67 & 83 & 65 & 94 & 89 & & 67 & 79 & 66 \\
\hline Wolfenden & 29 & 56 & 82 & & 80 & 47 & 76 & 64 & 97 & 90 & & 71 & 74 & 72 \\
\hline WW & 27 & 90 & 75 & & 81 & 83 & 59 & 60 & 79 & 89 & & 72 & 53 & 80 \\
\hline GES & 23 & 93 & 68 & & 78 & 87 & 53 & 58 & 95 & 89 & & 69 & 77 & 68 \\
\hline Eisenberg & 20 & 90 & 63 & & 72 & 89 & 47 & 56 & 93 & 86 & & 62 & 80 & 61 \\
\hline $\mathrm{KD}$ & 13 & 88 & 59 & & 63 & 91 & 42 & 54 & 95 & 91 & & 71 & 71 & 72 \\
\hline Heijne & 11 & 89 & 55 & & 51 & 91 & 35 & 52 & 93 & 83 & & 60 & 83 & 58 \\
\hline Hopp-Wodds & 11 & 87 & 58 & & 54 & 90 & 36 & 52 & 94 & 83 & & 58 & 83 & 58 \\
\hline Sweet & 11 & 87 & 59 & & 58 & 88 & 38 & 48 & 91 & 84 & & 59 & 80 & 58 \\
\hline Av-Cid & 10 & 87 & 58 & & 53 & 89 & 36 & 47 & 95 & 83 & & 58 & 80 & 56 \\
\hline Roseman & 9 & 89 & 56 & & 48 & 91 & 34 & 45 & 93 & 82 & & 61 & 85 & 58 \\
\hline Levitt & 9 & 88 & 56 & & 49 & 91 & 35 & 45 & 92 & 82 & & 55 & 85 & 55 \\
\hline Nakashima & 9 & 88 & 56 & & 50 & 90 & 35 & 43 & 90 & 83 & & 63 & 83 & 60 \\
\hline A-Cid & 8 & 87 & 57 & & 52 & 89 & 35 & 40 & 93 & 79 & & 56 & 85 & 55 \\
\hline Lawson & 8 & 86 & 57 & & 43 & 89 & 32 & 39 & 88 & 83 & & 60 & 84 & 58 \\
\hline Radzicka & 6 & 87 & 56 & & 41 & 91 & 32 & 36 & 92 & 80 & & 56 & 84 & 56 \\
\hline Bull-Breese & 6 & 86 & 56 & & 40 & 91 & 32 & 33 & 86 & 79 & & 55 & 84 & 54 \\
\hline EM & 5 & 89 & 56 & & 41 & 91 & 32 & 31 & 92 & 77 & & 57 & 85 & 55 \\
\hline Fauchere & 5 & 87 & 56 & & 43 & 91 & 33 & 28 & 43 & 62 & & 62 & 28 & 56 \\
\hline
\end{tabular}


which SVMtmh obtains the highest score for $Q_{2}$ at $86 \%$, compared to the second best methods at $80 \%$. Generally, SVMtmh performs $3 \%$ to $12 \%$ better for the highresolution set than for the low-resolution in terms of per-segment scores. Meanwhile, for per-residue scores, the accuracy for the high- and low-resolution data sets is similar in the range of $81 \%$ to $90 \%$. The shaded area in Table 3 denotes the four top-performing approaches, which are selected to further predict newly solved membrane protein structures (Section 3.7).

\subsection{Discrimination between soluble and membrane proteins}

To assess our method's ability to discriminate between soluble and membrane proteins, we apply SVMtmh to the soluble protein data set. A cut-off length is chosen as the minimum TMH length. Any protein that does not have at least one predicted TMH exceeding the minimum length is classified as a soluble protein. We calculate the false positives (FP) rates for the soluble protein set, where a false positive represents a soluble protein being falsely classified as a membrane protein. Similarly, we also calculate the false negatives $(\mathrm{FN})$ rates for both high- $\left(\mathrm{FN}_{\text {high }}\right)$ and low-resolution $\left(\mathrm{FN}_{\text {low }}\right)$ membrane protein sets using the chosen cut-off length. Clearly, the cut-off length is a trade-off between the FP and FN rates. Therefore, the cut-off length selected must minimize $\mathrm{FP}+\mathrm{FN}_{\text {high }}+\mathrm{FN}_{\text {low }}$. Fig. 4 shows the FP and FN rates as a function of cut-off length. The cut-off length at 18 , which minimizes the sum of all errors is used to discriminate between soluble and membrane proteins. Table 4 shows the results of our method compared to the other methods. SVMtmh is capable of distinguishing soluble and membrane proteins at $\mathrm{FP}$ and $\mathrm{FN}_{\text {low }}$ rates at less than $1 \%$ and $\mathrm{FN}_{\text {high }}$ rate at $5.6 \%$. In general, most advanced methods such as TMHMM $2^{3}$ and PHDpsiHtm08 ${ }^{12}$ achieve better accuracies than simple hydrophobicity scale methods including KyteDoolittle (KD) ${ }^{8}$ and White -Wimley (WW $)^{10}$.

\subsection{Effect of alternating geometric scoring function on topology ac- curacy}

We characterize the dependency of topology accuracy $(T O P O)$ on the values of the base $(b)$ and the exponent increment $(E I)$ used in the alternating geometric scoring function for the low-resolution data set. Fig. 5 shows

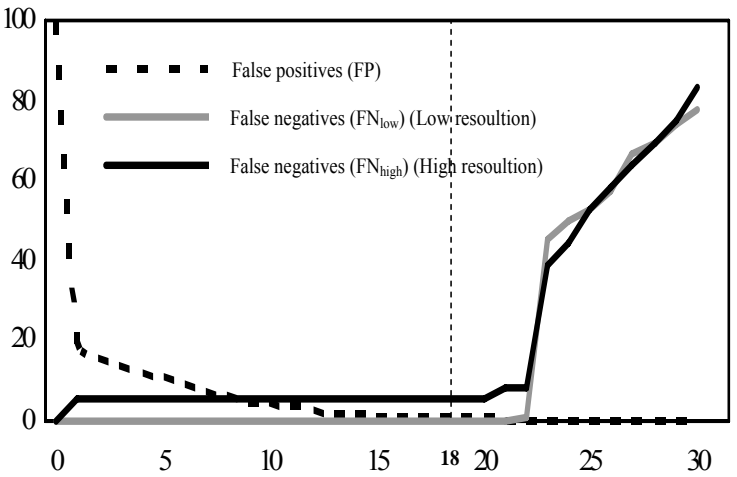

Fig. 4. The false positive and false negative rates as a function of cut-off length. The $\mathrm{x}$-axis: cut-off length; the $\mathrm{y}$-axis: false positive and false negative rates (\%). Discrimination between soluble proteins and membrane proteins is based on the cut-off length chosen. The cutoff length at 18 (dashed line) is chosen to minimize the sum of all three error rates $\left(\mathrm{FP}+\mathrm{FN}_{\text {low }}+\mathrm{FN}_{\text {high }}\right)$.

Table 4. Confusion between soluble and membrane proteins. The results of all compared methods are taken from Chen et al. ${ }^{13}$. False positive rates for soluble proteins are calculated in the second column In the third and fourth columns, false negative rates for membrane proteins are reported. Methods are sorted by false positive rates.

\begin{tabular}{lccc}
\hline \hline & \multirow{2}{*}{ False } & \multicolumn{2}{c}{ False negatives (\%) } \\
\cline { 3 - 4 } Methods & positives $(\%)$ & Low-resolution & High-resolution \\
\hline SVMtmh & $\mathbf{0 . 5}$ & $\mathbf{0}$ & $\mathbf{5 . 6}$ \\
TMHMM2 & 1 & 4 & 8 \\
SOSUI & 1 & 4 & 8 \\
PHDpsiHtm08 & 2 & 8 & 3 \\
PHDhtm08 & 2 & 23 & 19 \\
Wolfenden & 2 & 13 & 39 \\
Ben-Tal & 3 & 4 & 11 \\
PHDhtm07 & 3 & 16 & 14 \\
PRED-TMR & 4 & 1 & 8 \\
HMMTOP2 & 6 & 1 & 0 \\
TopPred2 & 10 & 11 & 8 \\
DAS & 16 & 0 & 0 \\
WW & 32 & 0 & 0 \\
GES & 53 & 0 & 0 \\
Eisenberg & 66 & 0 & 0 \\
KD & 81 & 0 & 0 \\
Sweet & 84 & 0 & 0 \\
Hopp-Woods & 89 & 0 & 0 \\
Nakashima & 90 & 0 & 0 \\
Heijne & 92 & 0 & 0 \\
Levitt & 93 & 0 & 0 \\
Roseman & 95 & 0 & 0 \\
A-Cid & 95 & 0 & 0 \\
Av-Cid & 95 & 0 & 0 \\
Lawson & 98 & 0 & 0 \\
FM & 99 & 0 & 0 \\
Fauchere & 99 & 0 & 0 \\
Bull-Breese & 100 & 0 & 0 \\
Radzicka & 100 & & 0 \\
\hline \hline & & 0 & 0 \\
\hline
\end{tabular}

the relationships between topology accuracy coded by colours and the variables in the scoring function. The white circles indicate the highest topology accuracy at about $84 \%$ and their corresponding values for $b$ and $E I$. The region in which half of the white circles $(8 / 16)$ occur falls in the ranges for $b$ and $E I$ between $[1.5,2.5]$ 
and $[0.5,1.5]$, respectively. The set of values for $(b, E I)$ we choose for the scoring function is $(1.6,1.0)$. An interesting observation is that low topology accuracy (80\%: blue and $79 \%$ : navy) occurs in the vertical-left, lower-horizontal, and upper-right regions. In the vertical-left $(b=1)$ and the lower-horizontal $(E I=0)$ regions, the scoring function is simplified to assigning an equal weight of 1 to all loop signals regardless of their distance from the N-terminus. Conversely, in the upperright region, when both $b$ and $E I$ are large, the scoring function assigns very small weights to the loop signals downstream of the N-terminus. The poor accuracy in the vertical-left and the lower-horizontal region is a result of considering the contribution of every signal in the loop segments equally. On the other hand, in the upper-right region, the poor performance is due the contribution from downstream signals made negligible by the scoring function. Therefore, our analysis supports the assumptions we have made about our scoring function: 1) topology formation is a result of contributing signals distributed along the protein sequence, particularly in the loop regions; and 2) the contribution of each downstream loop segment on the first loop segment is not equal and diminishes as a function of distance away from the $\mathrm{N}$-terminus. Our results suggest that the inclusion of both assumptions in modeling membrane protein topology is a key factor in achieving the best topology accuracy.

\subsection{Performance on newly solved structures and analysis of bacte- riorhodopsin}

To illustrate the performance of the top four methods on the high and low-resolution data sets as shown in Table 3 , we test four recently solved membrane protein structures not included in the training set. The results are shown in Table 5 . The best predicted protein is a photosynthetic reaction center protein (PDB ID: 1umx_L), for which all methods predict all helices correctly $\left(Q_{o k}=\right.$ $100 \%)$. On the other hand, only two methods are capable of predicting all the helices from a bacteriorhodopsin (bR) structure (PDB ID: 1 tn0_A) correctly $\left(Q_{o k}=\right.$ $100 \%$ ). In terms of topology prediction, most methods predict correctly for all four proteins. We devote our analysis to bR to illustrate that TMH prediction is by no means a trivial task and continuous development in this area is indispensable in advancing our understanding of membrane protein structures.

Fig. 6(a) displays the high-resolution structure of bR from PDB. Bacteriorhodopsin (bR) is a member of the rhodopsin family, which is characterized with seven distinct transmembrane helices that can be indexed from Helix A to G. Studies of synthetic peptides of each of the seven TM helices of bR have shown that Helix A to Helix E can form independently stable helices when inserted into a lipid bilayer ${ }^{26}$. However, Helix G does

Table 5. Performance of top four approaches shaded in Table $\mathbf{3}$ for newly solved membrane proteins. Proteins are indicated by their PDB codes and their observed topologies. Topology terms $\mathrm{N}_{\text {in }}$ : $\mathrm{N}$-terminal loop on the inside of membrane; $\mathrm{N}_{\text {out }}$ : N-terminal loop on the outside of membrane. PRED_TOPO: predicted topology.

\begin{tabular}{|c|c|c|c|c|c|c|c|c|}
\hline \multirow{2}{*}{ Protein (observed topology) } & \multirow{2}{*}{ Methods } & \multirow{2}{*}{ PRED_TOPO } & \multicolumn{3}{|c|}{ Per-segment $(\%)$} & \multicolumn{3}{|c|}{ Per-residue (\%) } \\
\hline & & & $Q_{o k}$ & $Q_{h t m}^{\% \text { obs }}$ & $Q_{h t m}^{\% p r d}$ & $Q_{2}$ & $Q_{2 T}^{\% \text { obs }}$ & $Q_{2 T}^{\% \text { prd }}$ \\
\hline \multirow{4}{*}{$1 \operatorname{tn} 0 \_\mathrm{A}\left(\mathrm{N}_{\mathrm{out}}\right)$} & SVMtmh & $\mathbf{N}_{\text {out }}$ & 100 & 100 & 100 & 85 & 84 & 94 \\
\hline & TMHMM2 & $\mathrm{N}_{\text {out }}$ & 0 & 86 & 100 & 71 & 68 & 87 \\
\hline & PHDpsiHtm08 & $\mathrm{N}_{\text {out }}$ & 0 & 71 & 100 & 76 & 77 & 87 \\
\hline & HMMTOP2 & $\mathrm{N}_{\text {out }}$ & 100 & 100 & 100 & 73 & 69 & 90 \\
\hline \multirow{4}{*}{$1 \mathrm{vfp} \_\mathrm{A}\left(\mathrm{N}_{\mathrm{in}}\right)$} & SVMtmh & $\mathbf{N}_{\text {in }}$ & $\mathbf{0}$ & 70 & 100 & 87 & 57 & 74 \\
\hline & TMHMM2 & $\mathrm{N}_{\text {in }}$ & 0 & 70 & 100 & 86 & 54 & 72 \\
\hline & PHDpsiHtm08 & $\mathrm{N}_{\text {in }}$ & 0 & 50 & 50 & 86 & 52 & 72 \\
\hline & HMMTOP2 & $\mathrm{N}_{\text {in }}$ & 0 & 80 & 89 & 85 & 58 & 63 \\
\hline \multirow{4}{*}{ lumx_L $\left(\mathrm{N}_{\text {in }}\right)$} & SVMtmh & $\mathbf{N}_{\text {in }}$ & 100 & 100 & 100 & 90 & 91 & 89 \\
\hline & TMHMM2 & $\mathrm{N}_{\text {in }}$ & 100 & 100 & 100 & 85 & 78 & 89 \\
\hline & PHDpsiHtm08 & $\mathrm{N}_{\text {out }}$ & 100 & 100 & 100 & 82 & 92 & 75 \\
\hline & HMMTOP2 & $\mathrm{N}_{\text {in }}$ & 100 & 100 & 100 & 83 & 78 & 83 \\
\hline \multirow{4}{*}{$1 \mathrm{xfh} \_\mathrm{A}\left(\mathrm{N}_{\mathrm{in}}\right)$} & SVMtmh & $\mathbf{N}_{\text {in }}$ & $\mathbf{0}$ & 70 & 78 & 60 & 62 & 60 \\
\hline & TMHMM2 & $\mathrm{N}_{\text {in }}$ & 0 & 70 & 88 & 63 & 57 & 65 \\
\hline & PHDpsiHtm08 & $\mathrm{N}_{\text {in }}$ & 0 & 50 & 56 & 53 & 69 & 53 \\
\hline & HMMTOP2 & $\mathrm{N}_{\text {in }}$ & 0 & 90 & 90 & 71 & 73 & 71 \\
\hline
\end{tabular}


40

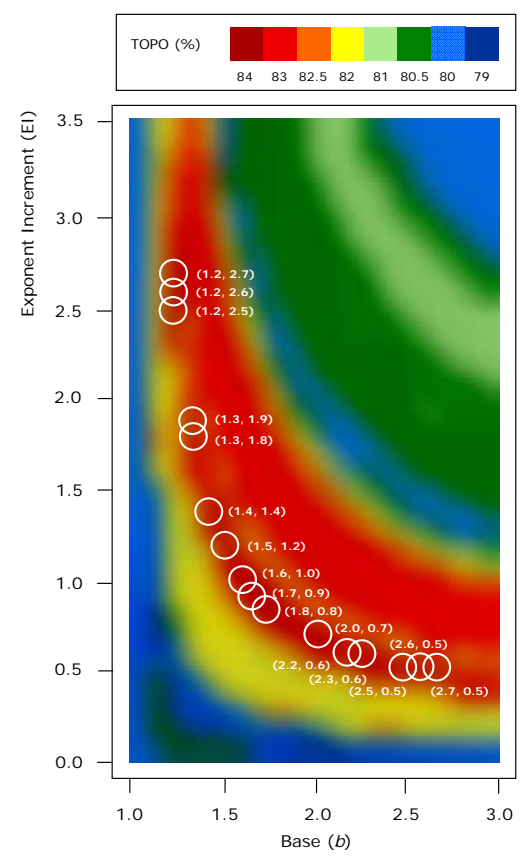

Fig. 5. The relationship between base $(b)$ and exponent increment $(E I)$ in the alternating geometric scoring function and topology accuracy. The x-axis: base $(b)$; the y-axis: exponent increment $(E I)$. The accuracy of topology prediction $(T O P O)$ for low-resolution data set is divided into 8 levels, each indicated by a colour. The best accuracy $(84 \%)$ and its associated $(b, E I)$ values occur within the white circles.

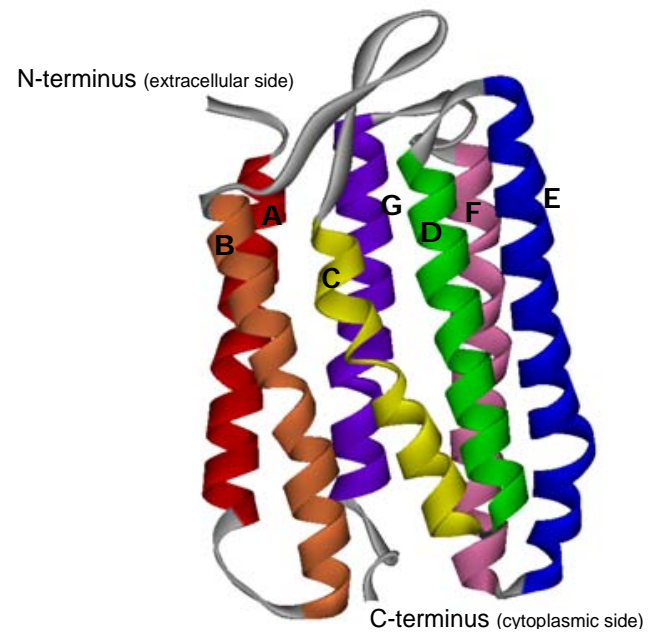

(a)

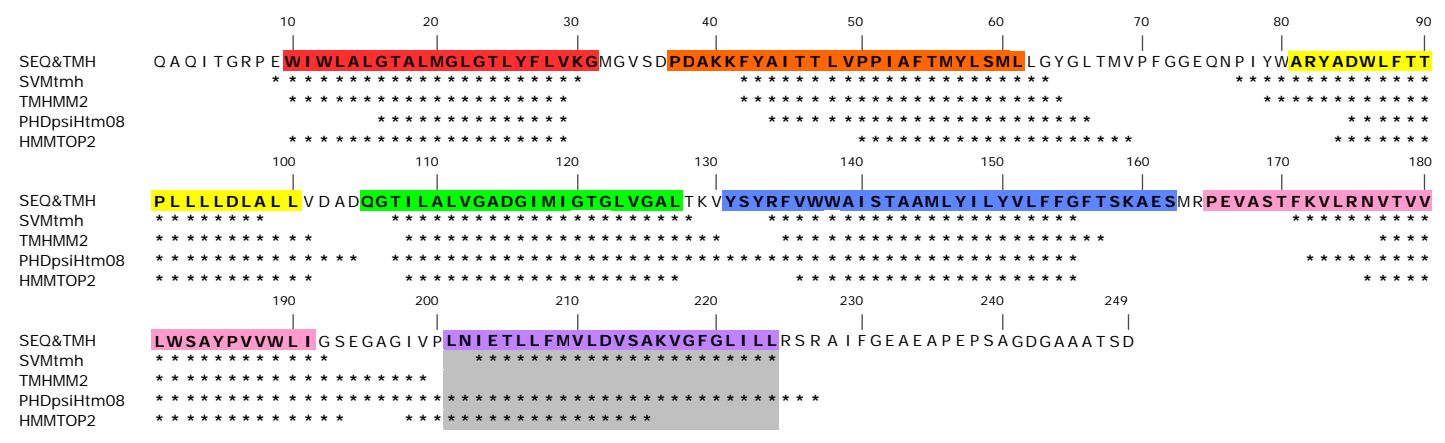

(b)

Fig. 6(a). The structure of a bacteriorhodopsin (bR) (PDB ID: 1tn0_A). Each helix is coloured and indexed from A to G. Figure is prepared with ViewerLite ${ }^{29}$. Fig. 6(b). Prediction results of bR by the top four methods $(*=$ predicted helix). The observed helices are indicated by colour boxes. The region of Helix G (purple) and its predictions are highlighted in grey. 
not form a stable helix in detergent micelles ${ }^{27}$ and exhibits structural irregularity at Lys 216 by forming a $\pi$ bulge $^{28}$. However, despite its atypical structure, Helix G is important in the function of $b R$, as it binds to retinal and undergoes conformation change during the photosynthetic cycle $^{28}$.

The results of the predictions by all four approaches are shown in Fig. 6(b). Interestingly, all approaches are successful in identifying the first six helices (Helix A - E) with good accuracy. However, most methods do not predict with the same level of success for Helix G. In particular, TMHMM2 misses Helix G entirely and PHDpsihtm08 merges predictions for Helix $F$ and Helix $G$ into one long helix. SVMtmh and HMMTOP $2^{11}$ are the only two out of all four methods that can correctly identify the presence of Helix G. Furthermore, upon a closer examination of Helix G, HMMTOP2 over-predicts by 3 residues at the Nterminus and severely under-predicts by 9 residues at the C-terminus. SVMtmh only under-predicts by 2 residues at the N-terminus of Helix G. The poor prediction results may be due to the intrinsic structural irregularity as described earlier, which adds another level of complexity into the TMH prediction problem. Despite the difficulties involved in predicting the correct location of Helix G, SVMtmh is successful in producing a prediction for the bR structure, which is in close agreement with the experimental approach. One possible reason for our success in this case could be the integration of multiple biological input features that encompass both global and local information for TMH prediction. TMHMM2 and HMMTOP2 rely solely on amino acid composition as sequence information, while PHDpsiHtm08 only uses sequence information from multiple sequence alignments. In contrast, SVMtmh incorporates a combination of both physico-chemical and sequence-based input features for helix prediction.

\section{CONCLUSION}

We have proposed an approach based on SVM in a hierarchical framework to predict transmembrane helix and topology in two successive steps. We demonstrate that by separating the prediction problem using two classifiers, specific biological input features associated with individual classifiers can be applied more effectively. By integrating both the sequence and structural input features and using a novel topology scoring function, SVMtmh achieves comparable or better persegment and topology accuracy for both high- and lowresolution data sets. When tested for confusion between membrane and soluble proteins, SVMtmh discriminates between them with the lowest false positive rate compared to the other methods. We further analyze a set of newly solved structures and show that SVMtmh is capable of predicting the correct helix and topology of bacteriorhodopsin as derived from a high resolution experiment.

With regard to future work, we will continue to enhance the performance of our approach by incorporating more relevant features in both stages of helix and topology prediction. We will also consider some complexities of TM helices, including helix lengths, tilts, and structural motifs, as in the case of bacteriorhodopsin. Supported by the results we achieved, our approach could prove valuable for genome-wide predictions to identify potential integral membrane proteins and their topologies.

While obtaining high-resolution structures for membrane proteins presents itself as a major challenge in the field of structural biology, the need for accurate prediction methods is highly demanded. We believe that the continuous development of computational methods with the integration of biological knowledge in this area will be immensely fruitful.

\section{Acknowledgments}

We gratefully thank Jia-Ming Chang, Hsin-Nan Lin, Wei-Neng Hung, and Wen-Chi Chou for providing helpful discussions and computational assistance. This work was supported in part by the thematic program of Academia Sinica under grant AS94B003 and AS95ASIA02.

\section{References}

1. Wallin E and von Heijne G. Genome-wide analysis of integral membrane proteins from eubacterial, archaean, and eukaryotic organisms. Protein Sci 1998; 7: 1029-1038.

2. Stevens TJ and Arkin IT. The effect of nucleotide bias upon the composition and prediction of transmembrane helices. Protein Sci 2000; 9: 505-511.

3. Krogh A, Larsson B, von Heijne G, and Sonnhammer EL. Predicting transmembrane protein topol- 
ogy with a hidden Markov model: application to complete genomes. J Mol Biol 2001; 305: 567-580.

4. Ubarretxena-Belandia I and Engelman DE. Helical membrane proteins: diversity of functions in the context of simple architecture. Curr Op in Struc Bio 2001; 11: 370-376.

5. White SH. The progress of membrane protein structure determination. Protein Sci 2004; 13: 1948-1949.

6. Berman HM, Westbrook J, Feng Z, Gilliland G, Bhat TN, Weissig H, Shindyalov IN, and Bourne PE. The Protein Data Bank. Nucleic Acids Res 2000; 28: 235-242.

7. van Geest M and Lolkema JS. Membrane topology and insertion of membrane proteins: search for topogenic signals. Microbiol Mol Biol Rev 2000; 64: 13-33.

8. Kyte J and Doolittle RF. A simple method for displaying the hydropathic character of a protein. $J$ Mol Biol 1982; 157: 105-132.

9. Eisenberg D, Weiss RM, and Terwilliger TC. The hydrophobic moment detects periodicity in protein hydrophobicity. Proc Natl Acad Sci U S A 1984; 81: 140-144.

10. White SH and Wimley WC. Membrane protein folding and stability: physical principles. Annu Rev Biophys Biomol Struct 1999; 28: 319-365.

11. Tusnady GE and Simon I. Principles governing amino acid composition of integral membrane proteins: application to topology prediction. J Mol Biol 1998; 283: 489-506.

12. Rost B, Fariselli P, and Casadio R. Topology prediction for helical transmembrane proteins at $86 \%$ accuracy. Protein Sci 1996; 5: 1704-1718.

13. Chen CP, Kernytsky A, and Rost B. Transmembrane helix predictions revisited. Protein Sci 2002; 11: $2774-2791$.

14. Chang CC and Lin CJ. LIBSVM: A library for support vector machines. Software available at http://www.csie.ntu.edu.tw/ cjlin/libsvm/.

15. von Heijne G. Membrane protein structure prediction. Hydrophobicity analysis and the positiveinside rule. J Mol Biol 1992; 225: 487-494.

16. Hessa T, Kim H, Bihlmaier K, Lundin C, Boekel J, Andersson H, Nilsson I, White SH, and von Heijne G. Recognition of transmembrane helices by the endoplasmic reticulum translocon. Nature 2005; 433: 377-381.

17. Mitaku S, Hirokawa T, and Tsuji T. Amphiphilicity index of polar amino acids as an aid in the characterization of amino acid preference at membranewater interfaces. Bioinformatics 2002; 18: 608-616.

18. Zhou H and Zhou Y. Predicting the topology of transmembrane helical proteins using mean burial propensity and a hidden-Markov-model-based method. Protein Sci 2003; 12: 1547-1555.

19. Jayasinghe S, Hristova K, and White SH. Energetics, stability, and prediction of transmembrane helices. J Mol Biol 2001; 312: 927-934.

20. Goder V and Spiess M. Topogenesis of membrane proteins: determinants and dynamics. FEBS Letters 2001; 504: 87-93.

21. Popot JL and Engelman DM. Membrane protein folding and oligomerization: the two-stage model. Biochemistry 1990; 29: 4031-4037.

22. Moller S, Kriventseva EV, and Apweiler R. A collection of well characterised integral membrane proteins. Bioinformatics 2000; 16: 1159-1160.

23. Bairoch B, Apweiler R. The SWISS-PROT protein sequence database: its relevance to human molecular medical research. J. Mol. Med. 1997; 5: 312316.

24. Cao B, Porollo A, Adamczak R, Jarrell M, and Meller J. Enhanced recognition of protein transmembrane domains with prediction-based structural profiles. Bioinformatics 2006; 22: 303-309.

25. Wu TF, Lin CJ, and Weng RC. Probability estimates for multi-class classification by pairwise coupling. JMLR 2004; 5: 975-1005.

26. Booth PJ. Unravelling the folding of bacteriorhodopsin. Biochim Biophys Acta 2000; 1460: 414.

27. Hunt JF, Earnest TN, Bousche O, Kalghatgi K, Reilly K, Horvath C, Rothschild KJ, and Engelman DM. A biophysical study of integral membrane protein folding. Biochemistry 1997; 36: 1515615176.

28. Luecke H, Schobert B, Richter HT, Cartailler JP, and Lanyi JK. Structure of bacteriorhodopsin at 1.55 A resolution. J Mol Biol 1999; 291: 899-911.

29. ViewerLite for molecular visualization. Software available at http://www.jaici.or.jp/sci/viewer.htm. 